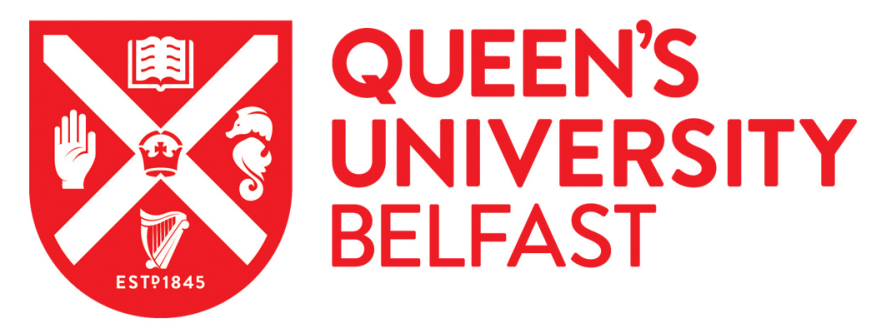

\title{
Interventions to optimise medication prescribing and adherence in older people with cancer: A systematic scoping review (protocol)
}

\author{
Murphy, M., Bennett, K., Hughes, C. M., Lavan, A., \& Cadogan, C. A. (2020). Interventions to optimise \\ medication prescribing and adherence in older people with cancer: A systematic scoping review (protocol). \\ Research in Social and Administrative Pharmacy. https://doi.org/10.1016/j.sapharm.2020.02.021
}

\section{Published in:}

Research in Social and Administrative Pharmacy

\section{Document Version:}

Peer reviewed version

Queen's University Belfast - Research Portal:

Link to publication record in Queen's University Belfast Research Portal

\section{Publisher rights}

Copyright 2020 Elsevier.

This manuscript is distributed under a Creative Commons Attribution-NonCommercial-NoDerivs License

(https://creativecommons.org/licenses/by-nc-nd/4.0/), which permits distribution and reproduction for non-commercial purposes, provided the author and source are cited.

\section{General rights}

Copyright for the publications made accessible via the Queen's University Belfast Research Portal is retained by the author(s) and / or other copyright owners and it is a condition of accessing these publications that users recognise and abide by the legal requirements associated with these rights.

Take down policy

The Research Portal is Queen's institutional repository that provides access to Queen's research output. Every effort has been made to ensure that content in the Research Portal does not infringe any person's rights, or applicable UK laws. If you discover content in the Research Portal that you believe breaches copyright or violates any law, please contact openaccess@qub.ac.uk. 
1 Interventions to optimise medication prescribing and adherence in older people with cancer:

2 A systematic scoping review (protocol)

3

4 Melanie Murphy $^{a}$, Kathleen Bennett ${ }^{\mathrm{b}}$, Carmel M. Hughes ${ }^{\mathrm{c}}$, Amanda Lavan $^{\mathrm{d}, \mathrm{e}}$, , Cathal A. Cadogan $^{\mathrm{a}}$ 5

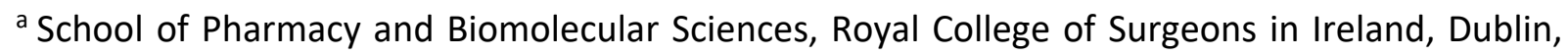
7 Ireland

$8{ }^{b}$ Population Health Sciences Division, Royal College of Surgeons in Ireland, Dublin, Ireland

9 'School of Pharmacy, Queen's University Belfast, Belfast, United Kingdom

$10{ }^{\mathrm{d}}$ Geriatric Medicine, Cork University Hospital, Cork, Ireland

11 e Department of Medicine for the Elderly, St James's Hospital, Dublin

12

13

14

15

16

17

18

19

20

21

Tel.: +35314025194

22

23

24

Corresponding author: Dr. Cathal Cadogan, School of Pharmacy and Biomolecular Sciences, Royal College of Surgeons in Ireland, 111 St. Stephen's Green, Dublin, Ireland

E-mail: cathalcadogan@rcsi.ie 
Background: Older adults with cancer often require multiple medications including cancerspecific treatments and supportive care medications (e.g. analgesics), as well as medications for pre-existing medical conditions. Increasing numbers of medications pose risks of potentially inappropriate prescribing, drug-drug interactions and drug-disease interactions. The burden of treatment (i.e. the workload of healthcare and its impact on patient functioning and well-being) may also negatively affect the way patients take their medications. Non-adherence to medication in patients with cancer is associated with treatment failure and increased healthcare costs. Therefore, it is crucial that medicines are optimised for older adults with cancer to enhance appropriate prescribing, reduce the complexity of treatment regimens and minimise the risk of non-adherence.

Objective: To provide an overview of evaluations of interventions aimed at optimising medication prescribing and/or adherence in older adults with cancer.

Methods: A systematic scoping review will be undertaken. Four databases will be searched from inception: PubMed, EMBASE, CINAHL and PsycINFO. In order to meet inclusion criteria, studies must evaluate an intervention seeking to improve medication prescribing and/or adherence in older adults (aged $\geq 65$ years) with an active cancer diagnosis using a comparative evaluation (e.g. inclusion of a control group). Two reviewers will independently screen titles and abstracts for inclusion and extract data relating to study population, intervention characteristics, outcome assessments and key findings. Extracted data will be collated using tables, figures and accompanying descriptive summaries. The review will be reported using the Preferred Reporting Items for Systematic reviews and Meta-Analyses extension for Scoping Reviews (PRISMA-ScR) guidelines.

Project impact: The scoping nature of this review will serve to provide an overview of the existing literature on interventions aimed at optimising medication prescribing and adherence in older adults with cancer. The review findings will help to identify research gaps and highlight areas to explore further in future research.

Key words: cancer, oncology, older adults, intervention, prescribing, adherence 


\section{Abbreviations:}

55 ADE: adverse drug event

56 EPOC: Effective Practice and Organisation of Care

57 PIP: potentially inappropriate prescribing

58 PRISMA-ScR: Preferred Reporting Items for Systematic Reviews and Meta-Analyses extension

59 for Scoping Reviews

60 RCT: randomised controlled trial

61 STOPP/START: Screening Tool of Older Person's Prescriptions / Screening Tool to Alert doctors

62 to Right Treatment

63

64 


\section{Introduction}

The population is ageing and by 2050 , it is predicted that $17 \%$ of the global population will consist of individuals aged $\geq 65$ years (conventionally described as "older") (1). Advancing age is a major risk factor for cancer (2). In 2012, 48\% of all cancer diagnoses worldwide occurred in older adults and, by 2038 , this is expected to rise to $58 \%$ (2). At diagnosis, older patients with cancer often have existing health conditions necessitating the use of polypharmacy, which is commonly defined as the prescribing of five or more medications (3-5). The reported prevalence of polypharmacy in older patients with cancer ranges from $13 \%$ to $92 \%$ (6). Polypharmacy contributes to patients' overall treatment burden (i.e. the workload of healthcare and its impact on patient functioning and well-being) (7). Treatment burden may be further increased by cancer-specific treatments and any additional supportive care medications (e.g. analgesics) that are required. Consequently, interventions aimed at optimising treatment regimens in cancer patients are required.

Medicines optimisation is an approach to care that aims to ensure the greatest clinical benefits for patients, through the safe and effective use of medicines (10). Medicines optimisation is individual to each patient and accounts for their specific needs, preferences and values (11). Optimising prescribing involves examining potentially inappropriate prescribing, as well as encouraging the appropriate use of medications in a way that the patient is willing to adhere to (12). The concept of medicines optimisation is particularly relevant to older adults as observational studies have highlighted associations between polypharmacy and potentially inappropriate prescribing (PIP), as well as negative clinical consequences, such as adverse drug events (ADEs) (8). This has also been observed in oncology settings, whereby a high proportion (21\%) of admissions to cancer services among older patients are related to adverse drug reactions arising from both the use of systemic anticancer treatments and non-cancer specific treatments (9).

The term PIP encompasses a range of suboptimal prescribing practices, including inappropriate doses or durations of medication, prescribing medications associated with high risks of ADEs and 
errors of omission (13). Various criteria have been developed and validated to assess and optimise medication prescribing in the general older population (14-17). These criteria can largely be classified as explicit (criterion-based, e.g. Screening Tool of Older Person's Prescriptions/Screening Tool to Alert doctors to Right Treatment (STOPP/START) $(17,18)$ ) or implicit (judgement-based, e.g. Medication Appropriateness Index (19)). Application of these criteria in clinical settings has been shown to improve the appropriateness of prescribing for older people (20). However, the applicability of these criteria to older patients with cancer is often limited, as prescribing for this patient cohort requires additional considerations, such as life expectancy and time until treatment benefit (21). In addition, these tools frequently recommend the cessation or avoidance of drugs that may be clinically appropriate for older adults with cancer (e.g. non-steroidal anti-inflammatory drugs can be of particular benefit for various forms of cancer pain) $(22,23)$. A deprescribing guideline has been developed that is specific to cancer populations at the palliative stage of illness (24). However, the extent of its clinical impact has yet to be determined.

The challenges that polypharmacy present are not limited to prescribing. Increasing numbers of medications may negatively impact the way in which patients take their medications. The World Health Organisation defines adherence as “the extent to which a person's behaviour-taking medication, following a diet, and/or executing lifestyle changes, corresponds with agreed recommendations from a healthcare provider" (25). Rates of medication adherence in patients with cancer vary widely, ranging from $46 \%$ to $100 \%$ (26). Previous studies have indicated that polypharmacy and complex medication regimens negatively impact on medication adherence $(25,27)$. Non-adherence to medication in patients with cancer has been associated with treatment failure, poor clinical outcomes, and increased healthcare costs $(28,29)$. Therefore, it is crucial that medicines are optimised in older adults with cancer to reduce the complexity of treatment regimens, minimise the risk of non-adherence and, ultimately, improve clinical outcomes. 
Several systematic reviews have examined interventions to improve the appropriate use of polypharmacy in older adults across a variety of settings $(13,20,30,31)$. However, none of these reviews have focussed specifically on older adults with cancer. For example, a Cochrane review on interventions to improve appropriate prescribing of polypharmacy for older people specifically excluded studies of interventions targeting patients with terminal illnesses and patients receiving chemotherapy (20). This has implications in terms of the generalisability of existing evidence to older patients with cancer. Furthermore, relatively few of the included studies (5/32 studies) assessed adherence as an outcome. A scoping review will be undertaken of interventions, evaluated to date, that have focussed on two key aspects of medicines optimisation for older people with cancer: appropriate prescribing and medication adherence. As the aim of this review is to provide a broad overview of the existing literature, a scoping review is preferable to a systematic review. Systematic reviews tend to be more specific in nature and normally focus on a more precise review question (32). Scoping reviews aim to identify and map the key concepts underpinning a research area (33). Additionally, they can help to identify research gaps and to highlight areas to explore further in future research (34). The objectives of this scoping review are to:

1. Identify the types of interventions aimed at optimising medication prescribing and/or adherence in older adults with cancer.

2. Characterise key features of the interventions (i.e. content, delivery, setting).

3. Examine the intervention development process (i.e. underpinning evidence and theory).

143 4. Characterise the study populations.

1445 . Identify the outcome measures that have been used to evaluate the interventions.

145 6. Establish key findings of the evaluations.

\section{Methods}

148 This review protocol has been developed in accordance with relevant methodological guidance

$149(34,35)$. The final review will be reported using the Preferred Reporting Items for Systematic Reviews and Meta-Analyses extension for scoping reviews (PRISMA-ScR) guidelines (36). 


\section{Search strategy}

153 Searches of the following electronic databases will be conducted using established search methods for scoping reviews: PubMed, EMBASE, CINAHL and PsycINFO (34). All databases will be searched from inception. The search strategy will comprise three stages:

1. Preliminary searches of each database will be conducted to identify relevant keywords and index terms.

2. A comprehensive search strategy for each electronic database will be developed with input from a research librarian.

3. Following database searching, reference lists of all included studies and any relevant review articles will be screened for additional studies.

162 The initial search terms will include combinations of terms such as: "cancer", "older adults", "prescribing" and "adherence", as well as relevant variants and synonyms. This will inform the development of a comprehensive search strategy. The full search strategy will be included in the final review manuscript.

\section{$\underline{\text { Inclusion criteria }}$}

169 Types of participants

170 This review will include studies involving older adults ( $\geq 65$ years) with an active cancer diagnosis.

171 Eligible studies will not be limited by the number or types of medicines prescribed, nor will they

172 be restricted to patients with any specific type of cancer or patients who are receiving treatment

173 in any particular healthcare setting. Studies which also involve other patient groups (e.g. patients

$174<65$ years, patients with other medical conditions) will be eligible for inclusion provided that data 175 for the subgroup of older cancer patients are available separately (either as published study data 176 or available separately from the study authors).

\section{$\underline{\text { Types of interventions }}$}


This review will include interventions seeking to optimise medication prescribing or adherence in older adults (aged $\geq 65$ years) with an active cancer diagnosis. Interventions must align with

181 key principles of medicines optimisation which will be broadly categorised as: interventions to 182 optimise prescribing and interventions to improve medication adherence (10). For the purpose 183 of this review, interventions to optimise prescribing will include interventions that target any of 184 the following: misprescribing (prescribing that significantly increases the risk of ADEs, including 185 incorrect dosing, frequency or duration), overprescribing (prescribing in the absence of a clinical 186 indication) and underprescribing (omission of medications for specific clinical indications aimed at either treatment or prevention) (37). Interventions aimed at improving medication adherence will include any intervention that seeks to promote adherence to prescribed medication in older patients with cancer. Eligible interventions can be targeted at the level of healthcare professional or patient. In order to meet inclusion criteria, interventions must specifically target medication prescribing or adherence in individuals aged $\geq 65$ years or a study population with a mean age of $\geq 65$ years.

\section{Types of outcome measures}

Due to the lack of an existing review of evaluations of interventions aimed at optimising medication prescribing and adherence in older adults with cancer, all outcomes for studies that meet the above inclusion criteria will be included in the review. This will allow for an overview to be provided of the range of outcomes that have been evaluated on this topic to date.

\section{$\underline{\text { Types of studies }}$}

201 In order to meet inclusion criteria, studies must have included some form of comparative 202 evaluation (e.g. inclusion of a control group or use of a controlled before/after design). . Only 203 full-text manuscripts published in English will be eligible for inclusion. Published conference abstracts will not be included in the review.

\section{Study selection}


Following deduplication, two reviewers will independently screen titles and abstracts for inclusion. If an abstract appears to meet inclusion criteria or cannot be excluded based on the title or abstract alone, the full-text article will subsequently be retrieved and assessed for inclusion by both reviewers independently. Any disagreements will be resolved through discussion and consultation with a third reviewer if necessary.

\section{Data extraction}

Two reviewers will independently perform data extraction (commonly referred to as 'charting the results' in the scoping review literature) using a data extraction form that has been developed in accordance with relevant methodological guidance (Appendix A) (34). The data extraction form will be piloted on one study that meets inclusion criteria and amended if necessary. Data will be extracted relating to each of the following:

1. Study: Authors, year of publication, country of origin, study design, study setting, study aims/purpose.

2. Population: Sample size, patient demographics (e.g. age, gender, type of cancer, stage of cancer, life expectancy, other medical conditions, numbers and types of medicines).

3. Intervention details: Intervention category (classified according to the Effective Practice and Organisation of Care (EPOC) taxonomy i.e. 'Delivery arrangements', 'Financial arrangements', 'Governance arrangements', 'Implementation strategies' (38)); intervention targets/recipients and deliverers, mode of intervention delivery, intervention duration, intervention costs/resource requirements.

4. Intervention development: details of any underpinning evidence base/theory base for the intervention.

5. Outcome measures used to evaluate the intervention: Reported outcomes, assessment methods and assessment time points.

6. Key findings: summary details of key results.

Any inconsistencies in terms of data extraction will be resolved through discussion and consultation with a third reviewer if necessary. 


\section{Data appraisal and synthesis}

239 As the aim of a scoping review is to provide a broad overview of the existing literature, formal 240 assessments of methodological quality of included studies are not routinely undertaken (34). Data collation will focus on summarising the information presented across included studies. A narrative synthesis will be undertaken in accordance with established guidance (40). This will 243 involve the following key steps (40):

244 (1) Developing a preliminary synthesis of findings of included studies: study characteristics and findings will be tabulated to summarise key study information and describe patterns across the studies in terms of direction and size of intervention effects.

(2) Exploring relationships in the data: extracted study data will be reviewed to identify factors that might help to explain differences in the direction and size of intervention effects across the included studies.

(3) Assessing the robustness of the synthesis: the findings of the synthesis process will be critically reviewed by the review team, in terms of the evidence on which it is based and potential sources of bias from the evidence sources, as well as any discrepancies and uncertainties identified relating to study outcome assessments. This process will help to

\section{Presentation of results}

In accordance with methodological guidance on scoping reviews (34), it is recommended that a proposed plan for presenting results should be developed at the time of protocol development. The findings of this review will be presented under the following headings to enable a logical and descriptive summary to be presented that aligns with the review's objectives:

- Search results

- Study designs

- Study aims/objectives

- Study populations 
- Interventions

- Outcomes recorded

- Key findings

270

271 A PRISMA-ScR flow diagram will be used to depict study selection. Tables of the characteristics

272 of included studies (Appendix B) and key study findings, together with accompanying descriptive summaries will be used to outline the data charted from included studies.

274

\section{Discussion}

276 Given the growing burden of cancer diagnoses among the older population, interventions 277 targeting medicines optimisation are of considerable importance in terms of ensuring 278 appropriate prescribing and use of medicines, and maximising their clinical benefits for patients.

279 This review will examine interventions aimed at optimising medication prescribing and/or 280 adherence in older adults with cancer in any healthcare setting. The scoping nature of the review 281 will serve to map existing literature on interventions that have been evaluated to date (e.g. in 282 terms of how interventions were developed, delivered and assessed). The review's findings will 283 lay important foundations in establishing current available evidence and informing future 284 research on developing and evaluating interventions to improve the care of older patients with 285 cancer in line with established methodological guidance (41).

\section{$\underline{\text { Acknowledgements }}$}

288 None

290 Conflicts of interest

291 None 
294 Melanie Murphy is supported by a Clement Archer Scholarship from the School of Pharmacy, 295 Royal College of Surgeons in Ireland.

296 Cathal Cadogan is jointly supported by the Irish Cancer Society and All Ireland Institute of Hospice 297 and Palliative Care [grant number: PAL17CAD].

298 Kathleen Bennett is supported by a HRB award [grant number:RL-15-1579].

299

300

301 


\section{$\underline{\text { References }}$}

303 1. He W, Goodkind D, Kowal P. U.S. Census Bureau, International Population Reports, 304 P95/16-1, An Aging World: 2015, U.S. Government Publishing Office, Washington, DC. 2016. 305 Available from:

306

307

\section{https://www.census.gov/content/dam/Census/library/publications/2016/demo/p95-16-1.pdf}

2. Pilleron S, Sarfati D, Janssen-Heijnen M, Vignat J, Ferlay J, Bray F, et al. Global cancer incidence in older adults, 2012 and 2035: A population-based study. International Journal of Cancer. 2019;144(1):49-58.

3. Deliens C, Deliens G, Filleul O, Pepersack T, Awada A, Piccart M, et al. Drugs prescribed for patients hospitalized in a geriatric oncology unit: Potentially inappropriate medications and impact of a clinical pharmacist. Journal of Geriatric Oncology. 2016;7(6):463-70.

4. Sharma M, Loh KP, Nightingale G, Mohile SG, Holmes HM. Polypharmacy and potentially inappropriate medication use in geriatric oncology. Journal of Geriatric Oncology. 2016;7(5):34653.

5. Masnoon N, Shakib S, Kalisch-Ellett L, Caughey GE. What is polypharmacy? A systematic review of definitions. BMC Geriatrics. 2017;17(1):230.

6. Maggiore RJ, Gross CP, Hurria A. Polypharmacy in older adults with cancer. Oncologist. 2010;15(5):507-22.

7. Eton DT, Ramalho de Oliveira D, Egginton JS, Ridgeway JL, Odell L, May CR, et al. Building a measurement framework of burden of treatment in complex patients with chronic conditions: a qualitative study. Patient Related Outcome Measures. 2012;3:39-49.

8. Maher RL, Hanlon J, Hajjar ER. Clinical consequences of polypharmacy in elderly. Expert Opinion on Drug Safety. 2014;13(1):57-65.

9. Lavan AH, O'Mahony D, Buckley M, O'Mahony D, Gallagher P. Adverse drug reactions in an oncological population: Prevalence, predictability, and preventability. Oncologist. 2019;24(9):e968-e977.

10. National Institute for Health and Care Excellence. NICE Guideline [NG5]: Medicines Optimisation: the safe and effective use of medicines to enable the best possible outcomes. United Kingdom; 2015. Available from: https://www.nice.org.uk/guidance/ng5. 
331 11. Cadogan CA, Ryan C, Hughes CM. Appropriate polypharmacy and medicine safety: When 332 many is not too many. Drug safety. 2016;39(2):109-16.

333 12. The King's Fund. Polypharmacy and medicines optimisation: making it safe and sound. 334 London; $2013 . \quad$ Available from:

335 http://www.kingsfund.org.uk/sites/files/kf/field/field_publication_file/polypharmacy-and-

336 medicines-optimisation-kingsfund-nov13.pdf. Accessed 05 May 2019.

337 13. Clyne B, Fitzgerald C, Quinlan A, Hardy C, Galvin R, Fahey T, et al. Interventions to address 338 potentially inappropriate prescribing in community-dwelling older adults: A systematic review of 339 randomized controlled trials. Journal of the American Geriatrics Society. 2016;64(6):1210-22.

340 14. O'Mahony D, O'Sullivan D, Byrne S, O'Connor MN, Ryan C, Gallagher P. STOPP/START 341 criteria for potentially inappropriate prescribing in older people: version 2. Age and Ageing. $342 \quad 2015 ; 44(2): 213-8$.

343 15. Lavan AH, Gallagher P, Parsons C, O'Mahony D. STOPPFrail (Screening Tool of Older 344 Persons Prescriptions in Frail adults with limited life expectancy): consensus validation. Age and 345 Ageing. 2017;46(4):600-7.

346 16. The 2019 American Geriatrics Society Beers Criteria ${ }^{\circledR}$ Update Expert Panel. American 347 Geriatrics Society 2019 Updated AGS Beers Criteria for Potentially Inappropriate Medication Use 348 in Older Adults. Journal of the American Geriatrics Society. 2019;67(4):674-694.

349 17. Gallagher P, Ryan C, Byrne S, Kennedy J, O'Mahony D. STOPP (Screening Tool of Older 350 Person's Prescriptions) and START (Screening Tool to Alert doctors to Right Treatment). 351 Consensus validation. International Journal of Clinical Pharmacology and Therapeutics. 352 2008;46(2):72-83.

353 18. Mahony DO, Sullivan DO, Byrne S, Connor MNO, Ryan C, Gallagher P. Corrigendum: 354 STOPP/START criteria for potentially inappropriate prescribing in older people: version 2. Age and 355 Ageing. 2018;47(3):489.

356 19. Samsa GP, Hanlon JT, Schmader KE, Weinberger M, Clipp EC, Uttech KM, et al. A 357 summated score for the medication appropriateness index: development and assessment of 358 clinimetric properties including content validity. Journal of Clinical Epidemiology. 1994;47(8):891359 6. 
20. Rankin A, Cadogan CA, Patterson SM, Kerse N, Cardwell CR, Bradley MC, et al. 361 Interventions to improve the appropriate use of polypharmacy for older people. The Cochrane 362 Database of Systematic Reviews. 2018(9):CD008165.

363 21. Holmes HM, Hayley DC, Alexander GC, Sachs GA. Reconsidering medication 364 appropriateness for patients late in life. Archives of Internal Medicine. 2006;166(6):605-9.

365 22. Whitman AM, DeGregory KA, Morris AL, Ramsdale EE. A comprehensive look at 366 polypharmacy and medication screening tools for the older cancer patient. Oncologist. $367 \quad 2016 ; 21(6): 723-30$.

368 23. Polidori P PC. Introducing Palliative Care 5th Edition. European Journal of Hospital 369 Pharmacy. 2017;24(131).

370 24. Lindsay J, Dooley M, Martin J, Fay M, Kearney A, Khatun M, et al. The development and 371 evaluation of an oncological palliative care deprescribing guideline: the 'OncPal deprescribing 372 guideline'. Supportive Care in Cancer. 2015;23(1):71-8.

373 25. Sabate E. World Health Organisation (WHO). Adherence to long-term therapies: evidence 374 for action. $2003 . \quad$ Available from: 375 https://www.who.int/chp/knowledge/publications/adherence_report/en/

376 26. Greer JA, Amoyal N, Nisotel L, Fishbein JN, MacDonald J, Stagl J, et al. A systematic review 377 of adherence to oral antineoplastic therapies. Oncologist. 2016;21(3):354-76.

378 27. Kardas P, Lewek P, Matyjaszczyk M. Determinants of patient adherence: a review of 379 systematic reviews. Frontiers in Pharmacology. 2013;4:91.

380 28. Neugut Al, Zhong X, Wright JD, Accordino M, Yang J, Hershman DL. Nonadherence to 381 medications for chronic conditions and non-adherence to adjuvant hormonal therapy in women 382 with breast cancer. JAMA Oncology. 2016;2(10):1326-32.

383 29. Partridge AH, Avorn J, Wang PS, Winer EP. Adherence to therapy with oral antineoplastic 384 agents. Journal of the National Cancer Institute. 2002;94(9):652-61.

385 30. Smith SM, Wallace E, O'Dowd T, Fortin M. Interventions for improving outcomes in 386 patients with multimorbidity in primary care and community settings. The Cochrane Database of 387 Systematic Reviews. 2016;3:CD006560. 

optimise prescribing in older people with dementia: A systematic review. Drugs \& Aging. 2019;36(3):247-67.

391 32. Munn Z, Peters MDJ, Stern C, Tufanaru C, McArthur A, Aromataris E. Systematic review or scoping review? Guidance for authors when choosing between a systematic or scoping review approach. BMC Medical Research Methodology. 2018;18(1):143.

394 33. Arksey H, O'Malley L. Scoping studies: towards a methodological framework.

395 International Journal of Social Research Methodology. 2005;8(1):19-32.

34. Peters MD, Godfrey CM, Khalil H, Mclnerney P, Parker D, Soares CB. Guidance for conducting systematic scoping reviews. International Journal of Evidence-based Healthcare. 2015;13(3):141-6.

35. Peters MDJ GC, Mclnerney P, Baldini Soares C, Khalil H, Parker D. Methodology for JBI scoping reviews. In: Adelaide AE, editor. The Joanna Briggs Institute reviewers' manual. Australia: The Joanna Briggs Institute; 2015. Available from: http://joannabriggs.org/assets/docs/sumari/reviewersmanual_methodology-for-jbi-scopingreviews_2015_v2.pdf.

36. Tricco AC, Lillie E, Zarin W, O'Brien KK, Colquhoun H, Levac D, et al. PRISMA Extension for Scoping Reviews (PRISMA-ScR): Checklist and Explanation. Annals of Internal Medicine. 2018; 169(7):467-73.

37. O'Connor MN, Gallagher P, O'Mahony D. Inappropriate prescribing: criteria, detection and prevention. Drugs \& Aging. 2012;29(6):437-52.

38. Effective Practice and Organisation of Care (EPOC). The EPOC taxonomy of health systems interventions. 2016. Available from: https://epoc.cochrane.org/resources/epoc-resources-review$\underline{\text { authors }}$

39. Higgins J, Altman DG SJe. Chapter 8: Assessing risk of bias in included studies. In: Higgins JPT, Green S (editors). Cochrane Handbook for Systematic Reviews of Interventions Version 5.1.0 (updated March 2011). The Cochrane Collaboration, 2011. Available from www.handbook.cochrane.org.

40. Ryan R; Cochrane Consumers and Communication Review Group. 'Cochrane 
417 Consumers and Communication Review Group: data synthesis and analysis'. 418 http://cccrg.cochrane.org, March 2019

419 41. Medical Research Council. Developing and evaluating complex interventions: New 420 guidance. London: Medical Research Council. 2008. Available from: 421 https://mrc.ukri.org/documents/pdf/complex-interventions-guidance/ 422

423 
424

425

426

427

428

429

430

431

432

433

434

435

436

437

438

439

440

441

442

443

444

445

446

447

448

449

450

451

452

\section{Appendices}

Appendix A: Data extraction form (outline)

Appendix B: Table of characteristics of included studies (key components)

\section{Appendix A: Data extraction form (outline)}

Data extraction fields

1. Author(s)

2. Year of publication

3. Country

4. Study design

5. Study aims/objectives

6. Study duration

7. Study population, setting and sample size

8. Intervention type

9. Details of intervention development

10. Assessment of intervention

11. Outcome measures

12. Results

Appendix B: Table of characteristics of included studies (key components)

1. Author, year of publication, country

2. Study design

3. Study duration

4. Study population, setting and sample size

5. Intervention type and comparison

6. Assessment of intervention

7. Outcome measures

8. Results 